\title{
Postural Behavior and Parkinson's Disease Severity
}

Paper accepted in TISHW 2016 published by IEEE being available in the IEEE Xplore Digital Library $\square$ and sent for indexing in ISI, Scopus, EI, INSPEC and Google Scholar.

\section{Catarina Godinho}

Centro de Investigação Interdisciplinar Egas Moniz (CiiEM), Instituto Superior de Ciências da Saúde Egas Moniz, Monte de Caparica, Portugal.

\section{José Brito}

Centro de Investigação Interdisciplinar Egas Moniz

(CiiEM), Instituto Superior de Ciências da Saúde Egas Moniz, Monte de Caparica, Portugal.

\section{Margarida Silva Dias}

Serviço de Neurologia do Centro Hospitalar de Lisboa Central, Protugal.
Véronique Ferret-Sena

Centro de Investigação Interdisciplinar Egas Moniz (CiiEM), Instituto Superior de Ciências da Saúde Egas Moniz, Monte de Caparica, Portugal.

Filipe Melo

Faculdade de Motricidade Humana, Universidade de Lisboa, Laboratório de Comportamento Motor

Estrada da Costa, 1499-002, Cruz Quebrada, Portugal

\section{INTRODUCTION}

Parkinson disease (PD) progression increases bradykinesia, tremor, gait disturbance and postural instability, which are the most severe problems interfering with patients' daily life activities and motor independence.

Freezing, falling, and ensuing events (such as fractures and others complications) increase morbidity and mortality of these patients [1, 2]. Reliable and valid measures related with Parkinson Disease impairments and functional limitations are important to characterize their capabilities and may be useful for the clinical practice and rehabilitation. With these measures, the progression of the disease and the effects of clinical intervention may be objectively assess and verifiable for clinical investigation [3][4]. Balance control can be assessed by observing standing upright posture, the ability to rise up from a chair, or the response to a push or pull at chest level (pulltest). Each of these tests is applied as part of the Unified Parkinson Disease Rating Scale (UPDRS or MDSUPDRS version). However, clinical scales present some degree of subjectivity. Several equipment and methodologies can be used to evaluate patients with balance disorders more objectively. Computerized Dynamic Posturography (CDP) is one of the most used in clinical evaluation of posture (Fig.1). while in stage IV, this projection showed a trend to change forwards. Concerning dynamic balance, LOS presents a reduction mainly in the forward direction in all stages. The directional control in anterior-posterior RWS decreases with disease progression.

Keywords-Postural Behavior; Computerized Dynamic Posturography; Parkinson Disease; clinical scales 


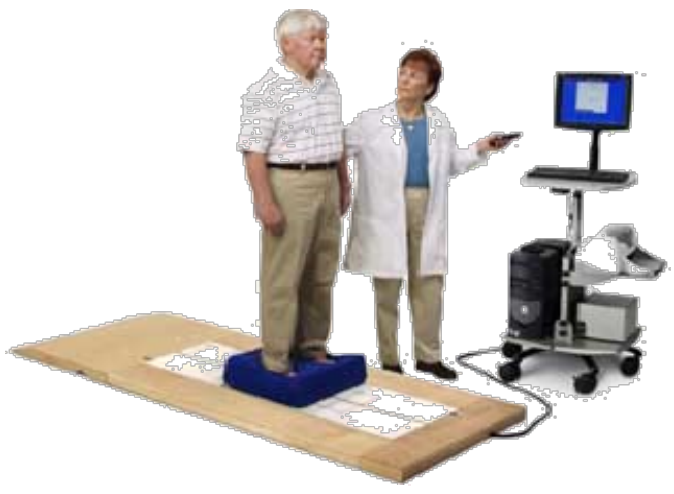

Fig 1. Computerized Dynamic Posturography using a Balance Master system

Quantitative analysis of balance and postural behavior, employing posturometric approaches, have been widely used to assess the progression of different types of intervention in PD patients, namely in pharmacotherapy [5], stereotactic surgeries $[6,4]$, physical activity and rehabilitation programs [7] or even in differential diagnosis [8]. CDP allows the detection of postural and strategic functional adaptations that might be unobserved during a normal clinical evaluation, like has been shown in several researches [9-11]. The aim of the present study was to identify and analyze quantitative data motor changes in the kinematic parameters, during static and dynamic postural tasks, according the levels of severity (stages) of PD patients. Postural behavior based on posturographic analysis of the center of pressure $(\mathrm{CoP})$ time series allowed to determine kinematic parameter(s) considered suitable to identify postural behavior modification in this population too.

\section{METHODS}

\section{A. Participants}

Our sample was composed of patients with the diagnosis of PD followed in clinical consultation at Hospital Santo António dos Capuchos, (Centro Hospitalar de Lisboa Central - EPE, Portugal). Participants with PD were excluded if they: 1) were classified in 0 or $\mathrm{V}$ in $\mathrm{H} \& \mathrm{Y}$ stages; 2) presented any other disease that could cause concomitant changes during walking or postural balance actions; and 3) had no cognitive capacity to follow the posturographic test protocol. The study was approved in 23/01/2007 by the local ethics committee of the Hospital Santo António dos Capuchos and conducted in accordance with the declaration of Helsinki. All subjects were given detailed information on the study and written informed consent to integrate the study was obtained.

\section{B. Clinical Assessments}

Patients selected in this research were all diagnosed with Parkinson Disease by a specialized neurologist in movement disorders, according to the UK brain bank criteria [12], and classificated in stages I to IV of the Hoehn and Yahr staging scale (H\&Y) [13]. The physician collected patient's demographics, clinical history, diagnosis, stage of the disease, daily living activities (UPDRS- Part II) [14] applied the pull test (question 30 of UPDRS-Part III), and the Schwab and
England Activities of Daily Living scale [15] and pharmacologic treatment were collected by the physician.

\section{Balance Assessments Balance Assessments}

Postural evaluation was performed with a Balance Master ${ }^{\circledR}$ system (Neurocom ${ }^{\circledR}$ International Inc., USA). The posturographic data were collected respecting an interval of 30 days since the clinical consultation. All testing was carried out about 1-2 $\mathrm{h}$ after the first morning drug administration to guarantee the "best on" clinical status.

The posturographic outcomes analysed were:

1) modified Clinical Test of Sensory Interaction on Balance (mCTSIB): this test quantifies the center of gravity $(\mathrm{CoG})$ postural sway velocity during four sensory conditions. The tests were performed in an upright stance during 30 seconds each (1- Eyes open on a firm surface (FirmEO); 2- Eyes closed on a firm surface (FirmEC); 3- Eyes open on an unstable surface (FoamEO); 4- Eyes closed on an unstable surface (FoamEC)) (Fig 2).

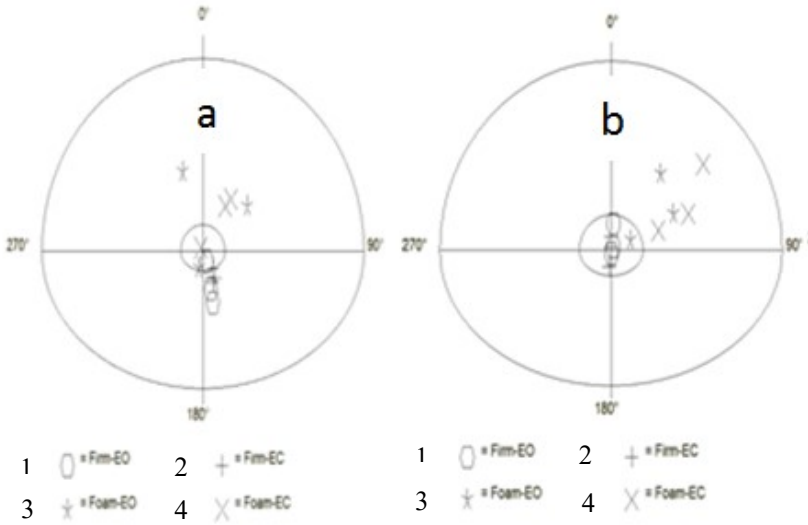

Fig 2. CoP alignment of PD patients in stage I (a) and stage III (b)

2 ) Limits of Stability (LOS): this test quantifies the reaction time and the maximal excursion of the CoP corresponding to the maximum distance a person can intentionally displace its CoP, i.e., lean their body in a given direction without losing balance, stepping or reaching for assistance (Fig 3).

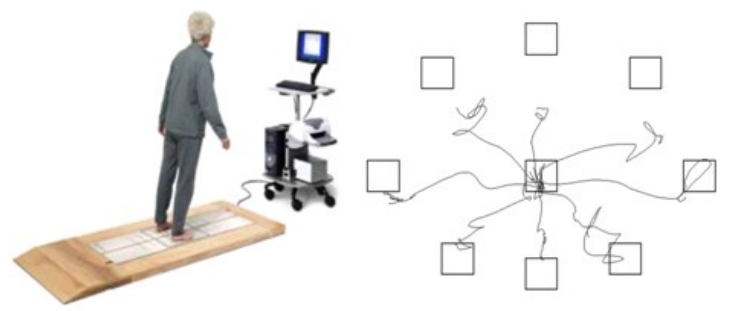

Fig 3. LOS during the displacement of the $\mathrm{CoP}$ in eight directions

3) Rhythmic Weight Shift (RWS): this test quantifies the CoP velocity and movement directional control of patient's ability to rhythmically lean their body (weight transfer) from left to right (medio-lateral direction) and forwards to backwards (anterior-posterior direction) between two targets 
at two distinct speeds: slow (3 s pacing) and medium $(2 \mathrm{~s}$ pacing) (Fig. 4).

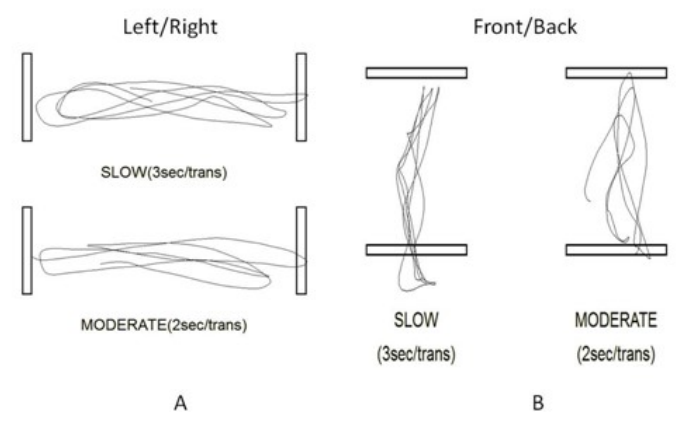

Fig 4. CoP trajectory of a PD patient, during medio-lateral (A) and anterior-posterior (B) rhythmic movements.

\section{STATISTICS}

The results were analyzed comparing the four groups of patients classified according to the H\&Y scale. Statistical analyses integrated a multivariate approach for the comparisons between groups, following the recommendation for a MANOVA one-way using the statistical test KeiserMeyer-Olkin (KMO). The normality tests were performed using Shapiro-Wilk test. For the homogeneity of variances, it was used the Levene test and for the homogeneity of variancecovariance matrix it was used the M-box test. In cases of violation of assumptions, it was used, for the homogeneity of variances, the Tamhane test, and for the homogeneity of matrix the Pillai trace. ANOVAs, followed by post-hoc tests were used when the KMO value was under 0.5. Factor Analysis to compare groups was also performed using the Quartimax factor rotation method. All tests were two-sided considering a significance level of 0.05 .

\section{RESULTS}

\section{A. Participant characteristics}

Our sample was composed of 103 PD patients (45 female and 58 male, $70.5 \pm 8.4$ years). After clinical evaluation they were divided according to the $H \& Y$ scale: 15 were in $H \& Y$ stage I; 33 in H\&Y II; 47 in H\&Y III; and 8 in H\&Y IV. Their demographic and PD related information is presented in tables 1 and 2.

TABLE 1 - Individual characterization. Mean values (M), standard deviation (SD), percentage related with the total sample.

\begin{tabular}{rccccc}
\hline & $\begin{array}{c}\text { H\&Y 1 } \\
(\mathbf{n}=15)\end{array}$ & $\begin{array}{c}\text { H\&Y 2 } \\
(\mathbf{n}=\mathbf{3 3})\end{array}$ & $\begin{array}{c}\text { H\&Y 3 } \\
(\mathbf{n}=\mathbf{4 7})\end{array}$ & $\begin{array}{c}\text { H\&Y 4 } \\
(\mathbf{n}=\mathbf{8})\end{array}$ & $\begin{array}{c}\text { Total } \\
(\mathbf{n}=\mathbf{1 0 3})\end{array}$ \\
\hline Gender & & & & \\
Female & $7(46.6 \%)$ & $14(42.4 \%)$ & $20(42.5 \%)$ & $4(50 \%)$ & $45(43.6 \%)$ \\
Male & $8(53.3 \%)$ & $19(57.5 \%)$ & $27(57.4 \%)$ & $4(50 \%)$ & $58(56.3 \%)$ \\
Age (years) & & & & & \\
M \pm SD & $73 \pm 5.2$ & $70.4 \pm 10.2$ & $70.3 \pm 8.2$ & $67.6 \pm 6.4$ & $70.5 \pm 8.4$ \\
Min. & 64 & 44 & 48 & 55 & 44 \\
Max. & 83 & 85 & 89 & 76 & 89 \\
Professional Status & & & & & $7.8 \%$ \\
Active & $6.7 \%$ & $6.1 \%$ & $10.6 \%$ & $0 \%$ & $92.2 \%$ \\
Retired & $93.3 \%$ & $93.9 \%$ & $89.4 \%$ & $100 \%$ & \\
\hline
\end{tabular}

TABLE 2 - PD characterization. Mean values (M), standard deviation (SD), percentage related with the total sample.

\begin{tabular}{|c|c|c|c|c|c|}
\hline & $\begin{array}{l}\text { H\&Y 1 } \\
(n=15)\end{array}$ & $\begin{array}{l}H \& Y 2 \\
(n=33)\end{array}$ & $\begin{array}{l}\text { H\&Y } 3 \\
(n=47)\end{array}$ & $\begin{array}{l}\text { H\&Y } 4 \\
(n=8)\end{array}$ & $\begin{array}{c}\text { Total } \\
(n=103)\end{array}$ \\
\hline \multicolumn{6}{|l|}{ Side of beginning } \\
\hline Right & $73,3 \%$ & $40,6 \%$ & $63 \%$ & $50 \%$ & $56,4 \%$ \\
\hline Left & $26,7 \%$ & $59,4 \%$ & $37 \%$ & $50 \%$ & $43,6 \%$ \\
\hline \multicolumn{6}{|l|}{ Form of manifestation } \\
\hline Tremor & $80 \%$ & $57,6 \%$ & $42,6 \%$ & $37,5 \%$ & $52,4 \%$ \\
\hline Akinetic & $20 \%$ & $42,4 \%$ & $57,4 \%$ & $62,5 \%$ & $47,6 \%$ \\
\hline \multicolumn{6}{|l|}{ Disease duration (years) } \\
\hline Min & 0 & 0 & 0 & 10 & 0 \\
\hline Max. & 7 & 39 & 29 & 30 & 39 \\
\hline $\mathrm{M} \pm \mathrm{SD}$ & $1 \pm 2,1$ & $2 \pm 7,2$ & $6 \pm 6,8$ & $12 \pm 7,6$ & $6,3 \pm 7,3$ \\
\hline
\end{tabular}

The score obtained in the UPDRS - Part II was $12.6 \pm 6.9$ (range: 0 and 30 points). Considering the scale of Schwab and England Activities of Daily Living, patients achieved an average percentage of $78 \%$ (100\%, representing the highest number corresponding to a best functional independency autonomy).

\section{B. Some Postural Behavior Outcomes}

\section{1) mCTSIB test}

The In the mCTSIB test, five common factors explaining $86.7 \%$ of the total variance of mean velocity were found. Of these, only the first factor that is clearly dominated by all variables showed significant differences between the 4 groups of the H\&Y $(p<.001)$. Thus, the mean velocity of the CoP is significantly different depending on the stage of disease severity for all conditions of equilibrium performed during this test (vision $\times 2$ / surface $\times 2$ ). The oscillation velocity increases in anterior-posterior (AP) and medio-lateral (ML) directions being greater in unstable condition compared to the stable surface condition.

For the alignment of the CoP, the factor analysis identified five independent axes explaining $76.4 \%$ of the variance observed. Each of these five factors was considered by ANOVA one way as a criterion for comparison between the 4 groups defined by H\&Y scale. This analysis detected significant differences between groups for the third factor extracted by factor analysis $(p=.037)$, which is dominated by variables relating to executions in unstable surface, with eyes open or closed, in the AP direction. The alignment of CoP is 
significantly altered, being projected backwards with disease progression, up to stage III and forward in stage IV. However, it was also observed a forward projection of the $\mathrm{CoP}$ within each H\&Y stage related with the increase of somatosensory complexity of the task (vision and proprioception constraints) In table 3 , are presented the results of the mean position center and in table 4 the mean velocity.

TABLE 3 - Position of CoP medio-lateral position (X) and antero-posterior position (Y), of the H\&Y (I-IV) groups in the 4 conditions (eyes open- EO, eyes close - EC, in a firm or foam surface) of the MCTSIB test.

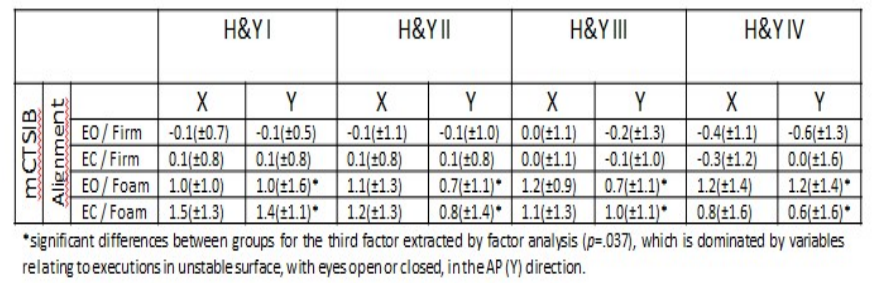

TABLE 4 - Velocity of CoP total (T), antero-posterior (AP) and medio-lateral (ML) displacement of the H\&Y (I-IV) groups in the 4 conditions (eyes openEO, eyes close - EC, in a firm or foam surface) of the mCTSIB test.

\begin{tabular}{|l|c|c|c|c|c|c|c|c|c|c|c|c|c|}
\hline \multicolumn{3}{|c|}{ H\&Y I } & \multicolumn{3}{|c|}{ H\&Y II } & \multicolumn{3}{|c|}{ H\&Y III } & \multicolumn{3}{|c|}{ H\&Y IV } \\
\hline & & T & AP & ML & T & AP & ML & T & AP & ML & T & AP & ML \\
\hline
\end{tabular}

\section{2) LOS test}

In the LOS test, seven common factors explaining $72.4 \%$ of the variance observed, were identified. The univariate model applied didn't detect significant differences between the 4 H\&Y stages, although these differences are suggested for the second factor extracted $(\mathrm{p}=.057)$, associated with point of excursion, maximal excursion and directional control parameters in the forward direction (Table 5).

TABLE 5. Mean and SD of LOS test parameters in 4 directions

\begin{tabular}{|c|c|c|c|c|c|c|}
\hline & & & H\&Y I & $H \& Y I I$ & $\mathrm{H} \& Y$ III & H\&Y IV \\
\hline \multirow{20}{*}{ 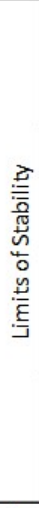 } & \multirow{5}{*}{ Forward } & Reaction time (s) & $1.2 \pm 0.6$ & $1.8 \pm 0.8$ & $1.6 \pm 1.0$ & $1.0 \pm 0.7$ \\
\hline & & Velocity $(\% / 5)$ & $2.0 \pm 1.4$ & $1.4 \pm 0.8$ & $1.4 \pm 0.8$ & $2.3 \pm 1.8$ \\
\hline & & Maximal excursion (\%) & $55 \pm 18$ & $54 \pm 22$ & $45 \pm 18$ & $50 \pm 17$ \\
\hline & & End point of excursion (\%) & $39 \pm 16$ & $40 \pm 22$ & $35 \pm 16$ & $33 \pm 10$ \\
\hline & & Directional control (\%) & $76 \pm 13$ & $76 \pm 18$ & $71 \pm 20$ & $59 \pm 28$ \\
\hline & \multirow{5}{*}{ Backward } & Reaction time (s) & $1.3 \pm 0.5$ & $1.0 \pm 0.5$ & $1.0 \pm 0.7$ & $0.6 \pm 0.2$ \\
\hline & & Velocity (o/s) & $1.5 \pm 1.0$ & $1.4 \pm 0.7$ & $1.3 \pm 0.7$ & $2.0 \pm 0.6$ \\
\hline & & Maximal excursion (\%) & $59 \pm 22$ & $59 \pm 18$ & $52 \pm 21$ & $57 \pm 19$ \\
\hline & & End point of excursion (\%) & $38 \pm 17$ & $41 \pm 11$ & $38 \pm 19$ & $39 \pm 15$ \\
\hline & & Directional control $(\%)$ & $65 \pm 21$ & $60 \pm 27$ & $59 \pm 27$ & $54 \pm 32$ \\
\hline & \multirow{5}{*}{ Right } & Reaction time (s) & $1.6 \pm 0.7$ & $1.5 \pm 0.5$ & $1.8 \pm 1.1$ & $0.8 \pm 0.5$ \\
\hline & & Velocity $(\because / 5)$ & $3.2 \pm 1.2$ & $2.7 \pm 1.0$ & $2.4 \pm 1.6$ & $4.3 \pm 2.5$ \\
\hline & & Maximal excursion (\%) & $79 \pm 19$ & $77 \pm 17$ & $68 \pm 24$ & $84 \pm 18$ \\
\hline & & End point of excursion (\%) & $65 \pm 24$ & $61 \pm 20$ & $53 \pm 26$ & $65 \pm 21$ \\
\hline & & Directional control (\%) & $75 \pm 11$ & $82 \pm 9$ & $75 \pm 16$ & $67 \pm 20$ \\
\hline & \multirow{5}{*}{ Left } & Reaction time (s) & $1.3 \pm 0.6$ & $1.6 \pm 0.9$ & $1.8 \pm 1.1$ & $1.2 \pm 0.9$ \\
\hline & & Velocity (o/s) & $3.5 \pm 1.3$ & $3.1 \pm 1.9$ & $2.5 \pm 1.6$ & $4.8 \pm 3.2$ \\
\hline & & Maximal excursion (\%) & $73 \pm 14$ & $76 \pm 15$ & $70 \pm 16$ & $73 \pm 22$ \\
\hline & & End point of excursion (\%) & $55 \pm 17$ & $62 \pm 18$ & $58 \pm 17$ & $54 \pm 21$ \\
\hline & & Directional control (\%) & $82 \pm 6,5$ & $80 \pm 9,6$ & $80 \pm 10$ & $75 \pm 22$ \\
\hline
\end{tabular}

The univariate model applied didn't detect significant differences between the 4 H\&Y stages, although these differences are suggested for the second factor extracted $(p=057)$, associated with end point excursion, maximal excursion and directional control parameters in the forward direction.

\section{3) RWS test}

In the RWS test, we observed a significant decrease in AP directional control during body intentional oscillation (CoP displacement), depending, for both speeds, on the disease progression. (Table 6). Two common factors explaining $64 \%$ of the observed variance, were identified. The univariate model allows to detect significant differences between the $4 \mathrm{H} \& \mathrm{Y}$ stages for the second common factor $(p=.04)$, associated to the parameters related to AP directional control in both rhythmic movement conditions ( 2 and 3 secunds). There was no difference regarding directional control or movement velocity in ML displacements, concerning task speed.

TABLE 6. Mean and SD of the RWS parameters

\begin{tabular}{|c|c|c|c|c|c|c|c|c|c|c|}
\hline & & & \multicolumn{2}{|c|}{$\mathrm{H} \& \mathrm{YI}$} & \multicolumn{2}{|c|}{$H \& Y$ II } & \multicolumn{2}{|c|}{ H\&Y III } & \multicolumn{2}{|c|}{ H\&YIV } \\
\hline & & & AP & $\mathrm{ML}$ & AP & $\mathrm{ML}$ & AP & $\mathrm{ML}$ & $A P$ & $\mathrm{ML}$ \\
\hline \multirow{4}{*}{$\sum_{x}^{u}$} & \multirow{2}{*}{\begin{tabular}{|c|} 
Slow speeds \\
(3s)
\end{tabular}} & Velocity $(0 / 5)$ & $1.6 \pm 0.4$ & $2.8 \pm 0.5$ & $1.5 \pm 0.4$ & $2.6 \pm 0.8$ & $1.3 \pm 0.5$ & $2.2 \pm 0.9$ & $1.7 \pm 0.5$ & $3.4 \pm 1.8$ \\
\hline & & Directional control (\%) & $59 \pm 11^{*}$ & $68 \pm 10$ & $68 \pm 15^{*}$ & $67 \pm 12$ & $50 \pm 24^{*}$ & $62 \pm 14$ & $40 \pm 26^{*}$ & $65 \pm 13$ \\
\hline & \multirow{2}{*}{$\begin{array}{c}\text { Medium speed } \\
\text { (2s) }\end{array}$} & Velocity $(\% / 5)$ & $2.2 \pm 0.8$ & $3.6 \pm 0.9$ & $2.1 \pm 0.6$ & $3.9 \pm 1.1$ & $1.9 \pm 0.8$ & $3.7 \pm 1.2$ & $2.2 \pm 1.0$ & $4.9 \pm 1.9$ \\
\hline & & Directional control (\%) & $65 \pm 14^{*}$ & $74 \pm 8$ & $70 \pm 12^{*}$ & $75 \pm 8$ & $55 \pm 26^{*}$ & $72 \pm 9$ & $38 \pm 31^{*}$ & $71 \pm 6$ \\
\hline
\end{tabular}

\section{DISCUSSION}

The present study pretended to analyze and quantify objectively the characteristic motor changes (kinematic parameters) related to different stages of Parkinson's disease progression. Goetz et all [16] verified that stage I and stage V account for the smallest number of subjects, followed by stage $\mathrm{IV}$, and the bulk of patients, ranging from 52 to $77 \%$, fall into stages II or III. In our study we found a similar distribution: stages I and IV have a smaller number of patients $(22,4 \%)$ followed by stages II and III (77, 6\%).

The Computerized Dynamic Posturography (CDP) has already proven to be useful for balance evaluation of PD patients $[10,17]$ giving insights into the physiological correlates of postural stability, [18] and gait [19]. Different studies refer that quantitative measures are not correlated with other clinical scores listed (e.g., duration of the disease and UPDRS) [20], or weakly correlated with H\&Y stages, [17]. Several studies have analyzed the postural sway characteristics of PD patients compared with age-matched controls $[21,18$, 22], nevertheless changes of the posturographic parameters as a function of the disease severity have not been very well studied. In this study, we focused our attention on the analysis of the variation of postural behavior parameters extracted from CoP time series presented at different stages established by clinical scales.

In the mCTSIB test we found that the mean velocity of oscillation increased with disease progression and this increase was greater when the test was performed with eyes closed. Blaszczyk et al. $[23,21]$ had already verified that the exclusion of visual information resulted in a decline in postural stability evidenced by an increase of the anterior-posterior sway range. The loss of visual input has been shown to improve an increase of muscle stiffness in most subjects related to postural compensating mechanism under eyes closed conditions [24]. This result is in agreement with the impairment verified, in parkinsonian populations [25]. Our results about CoP alignment are in agreement with the studies of Blaszczyk [23] and Schieppati [26] that analyzed the changes related to PD progression, attesting, under static conditions, a significant shift in the position of $\mathrm{CoP}$ related with disease severity. These 
authors also observed that in less affected patients (H\&Y I), the $\mathrm{CoP}$ was shifted backwards, and in the most severely affected patients (H\&Y III), the CoP was shifted forward as a consequence of a more stooped position. These previous studies reported an impaired ability of PD patients to lean forwards and backwards in voluntary tasks $[18,27]$. The visual information is not critical to the maintenance of balance during standing, it assumes greater importance with balance task complexity, or sensory input manipulation [28], as can been found with the use of unstable surfaces (foam). In our study, we observed the importance of the interaction between visual and proprioceptive systems through a significant change in the parameters related to postural sway during the different test conditions (open/closed eyes, stable/unstable surface). According to our results, the loss of visual information has a greater effect on variables related to postural tasks in unstable balance, due to changes in the available sources of sensory information (vision vs proprioception). This information may be useful in the context of developing rehabilitation programs by guiding the selection of movement complexity of the balance training programs according to stage severity.

Multidirectional posturography studies have also found that PD patients present reduced limits of stability $[10,20]$. The stooped posture typically observed in PD patients contributed to impair forward leaning $[29,26]$ and the reduced leg strength and power found in these patients [30] may contribute to limited performance during voluntary displacement of $\mathrm{CoP}$. In our study, the limits of stability (LOS) in the forward direction show a decrease with disease progression, although it is only observed a tendency of significant differences that must be confirm. This result was also observed in studies comparing PD patients in early versus late stages [23, 31]. However, it should be noted that these results may be related to those observed in the mCTSIB test where changes of the CoP alignment are associated with forward displacement as the disease severity progresses. As in our study, Suarez [32] observed also a decrease in the limits of stability of early stages when compared with a control group. Jessop et al. [33] observed also a decrease of the maximum excursion amplitude of the $\mathrm{CoP}$ displacement in individuals with mild to moderate PD stages, when compared to a control group. The authors found also that PD patients improved the capacity of increasing maximum excursion amplitudes after undergoing a program of physical activity.

The results of the Rhythmic Weight Shift (RWS) test show a significant decrease in movement directional control on the AP direction during intentional displacements of CoP, which is related with the severity progression. No differences between the 4 groups of disease severity during rhythmic ML movements were observed.

The amplitude of the CoP displacements may be influenced by the stiffness of the musculoskeletal system [34]. Previous studies have reported increased stiffness in PD patients [35, 20]. This increase helps to resist external perturbations, reducing the amplitude of the body oscillations, and preventing the generation of fast and reactive forces [36].

Rossi [17], in a similar study, observed that while movement velocity was lower in the group with $\mathrm{PD}$, there were no significant differences in the directional control parameter between PD patients and healthy control, which do not agree with our results. Beckley et al. [37], applying also a RWS test observed a decrease in the amplitude and movement velocity values of early stages PD patients when compared with a healthy control group.

The deterioration of balance, implicit in postural instability of PD patients, can be related with a loss of ability to intentionally control the CoP movement when performing activities involving weight transfer [26].

\section{CONCLUSIONS}

Individuals with PD characterized according to their stage of severity can be distinguished by static posturography parameters, namely the sway velocity which increases with disease severity. In early to medium stages of the disease, patients present a preferential projection backwards, while in most severe stages body projection is forwards. The total distance, the amplitude and the frequency of body sway increase significantly with disease severity and also with the sensory complexity of the tasks involved.

The area of support corresponding to the limits of stability presents a reduction in the movement amplitude mainly in the forward direction, causing a significant impairment in rhythmic movements implying body weight transfer in AP direction. This decrease is related with disease progression.

Our study showed that it is possible to identify differences in motor parameters between the four H\&Y stages of severity of PD patients, using a quantitative posturographic approach based on a set of variables associated with postural control. This more objective evaluation based on quantitative data may contribute for a better clinical survey of PD patients in terms of drug effects and fall prevention rehabilitation programs.

\section{Acknowledgements}

We are thankful to the collaboration of all patients who participated in this study and for all the support and collaboration of neurologists and staff members from Hospital Santo António dos Capuchos.

\section{REFERENCES}

[1] Hiorth YH, Lode K, Larsen JP . Frequencies of falls and associated features at different stages of Parkinson's disease. European journal of neurology, 2013, 20, 1: 160-166

[2] García Ruiz, J; Meseguer, E; Del Val, J; Vázquez, A; Bernardos, VS Vázquez, A. Motor Complications in Parkinson Disease: A prospective follow-up study. Clinical Neuropharmacology, 2004; 27, 2: 49-52.

[3] Sherer, T. B. Biomarkers for Parkinson's disease. Sci Transl Med, 2011; 3(79): $79 \mathrm{ps} 14$.

[4] Visser, J. E., Allum, J. H., Carpenter, M. G., Esselink, R. A., Speelman, J. D., Borm, G.F., et al. Subthalamic nucleus stimulation and levodoparesistant postural instability in Parkinson's disease. J Neurol, 2008; 255(2): 205-210

[5] Maurer, C., Mergner, T., \& Peterka, R. J. Abnormal resonance behavior of the postural control loop in Parkinson's disease. Exp Brain Res, 2004; 157(3): 369-376

[6] Colnat-Coulbois, S., Gauchard, G. C., Maillard, L., Barroche, G., Vespignani, H., Auque, J., et al. Bilateral subthalamic nucleus stimulation improves balance control in Parkinson's disease. J Neurol Neurosurg Psychiatry, 2005; 76(6): 780-787. 
[7] Qutubuddin, A. A., Pegg, P. O., Cifu, D. X., Brown, R., McNamee, S., \& Carne, W. Validating the Berg Balance Scale for patients with Parkinson's disease: a key to rehabilitation evaluation. Arch Phys Med Rehabil, 2005; 86(4): 789-792.

[8] Bloem, B. R. Postural Instability in Parkinson's disease, Clinical Neurology \& Neurosurgery, 1992; 94, S: 41-45.

[9] Bloem, B. R., van Vugt, J. P., \& Beckley, D. J. Postural instability and falls in Parkinson's disease. Adv Neurol, 2001; 87: 209-223.

[10] [Rossi-Izquierdo M, Basta D, Rubio-Rodríguez JP, Santos-Pérez S, Ernst A, Sesar-Ignacio A, Alberte-Woodward M, Guijarro-Del Amo M, Estany-Gestal A, San Román-Rodríguez E, Faraldo-García A, Zubizarreta-Gutiérrez A, Soto-Varela A. Is posturography able to identify fallers in patients with parkinson's disease? Gait \& Posture, 2014; 40, 1: 53-57.

[11] Visser, J. E., Carpenter, M. G., van der Kooij, H., \& Bloem, B. R. The clinical utility of posturography. Clin Neurophysiol, 2008; 119(11): 2424-2436.

[12] Hughes, A. J., Ben-Shlomo, Y., Daniel, S. E., \& Lees, A. J. What features improve the accuracy of clinical diagnosis in Parkinson's disease: a clinicopathologic study. Neurology, 1992; 42(6): 1142-1146.

[13] Hoehn, M. M., \& Yahr, M. D. Parkinsonism: onset, progression and mortality. Neurology, 1967; 17(5): 427-442.

[14] Fahn, S., \& Elton, R. UPDRS program members Parkinson's Disease Rating Scale. In F. S, M. CD, G. M \& C. DB (Eds.), Recent developments in Parkinson's disease, 1987; 2: 153-163. Florham Park: Macmillan Healthcare Information.

[15] Schwab, R. S., \& England, A. C. J. Projection technique for evaluating surgery in Parkinson's disease. In F. D. Gillingham, IML., (Ed.), Third Symposium on Parkinson's Disease. Livingstone; Edinburgh, Scotland. 1969. p 152-157.

[16] Goetz, C. G., Poewe, W., Rascol, O., Sampaio, C., Stebbins, G. T., Counsell, C., et al. (2004). Movement Disorder Society Task Force report on the Hoehn and Yahr staging scale: status and recommendations. Mov Disord. 2004; 19, 9: 1020-1028.

[17] Rossi, M., Soto, A., Santos, S., Sesar, A., \& Labella, T. A prospective study of alterations in balance among patients with Parkinson's Disease. Protocol of the postural evaluation. Eur Neurol. 2009; 61, 3: 171-176.

[18] Ganesan, M., Pal, P. K., Gupta, A., \& Sathyaprabha, T. N. Dynamic posturography in evaluation of balance in patients of Parkinson's disease with normal pull test: concept of a diagonal pull test. Parkinsonism Relat Disord. 2010; 16, 9: 595-599.

[19] Buckley, T. A., Pitsikoulis, C., \& Hass, C. J. Dynamic postural stability during sit-to-walk transitions in Parkinson disease patients. Mov Disord. 2008; 23, 9: 1274-1280.

[20] Horak, F. B., Dimitrova, D., \& Nutt, J. G. Direction-specific postural instability in subjects with Parkinson's disease. Exp Neurol. 2005; 193, 2: 504-521.

[21] Blaszczyk, J. W., \& Orawiec, R. Assessment of postural control in patients with Parkinson's disease: Sway ratio analysis. Hum Mov Sci. 2010; 30, 2: 396-404.
[22] Visser, J. E., Oude Nijhuis, L. B., Janssen, L., Bastiaanse, C. M., Borm, G. F., Duysens, J., et al. Dynamic posturography in Parkinson's disease: diagnostic utility of the "first trial effect". Neuroscience. 2010; 168, 2: 387-394.

[23] Blaszczyk, J. W., Orawiec, R., Duda-Klodowska, D., \& Opala, G. Assessment of postural instability in patients with Parkinson's disease. Exp Brain Res. 2007; 183, 1: 107-114.

[24] Jancova J. Measuring the balance control system - Review , Acta Medica. 2008; 51, 3: 129-137

[25] Bosek, M., Grzegorzewski, B., Kowalczyk, A., \& Lubinski, I. Degradation of postural control system as a consequence of Parkinson's disease and ageing. Neurosci Lett. 2005; 376, 3: 215-220.

[26] Schieppati, M., \& Nardone, A. (1991). Free and supported stance in Parkinson's disease. The effect of posture and 'postural set' on leg muscle responses to perturbation, and its relation to the severity of the disease. Brain. 1991; 114, 3: 1227-1244.

[27] Menant, J. C., Latt, M. D., Menz, H. B., Fung, V. S., \& Lord, S. R. Postural sway approaches center of mass stability limits in Parkinson's disease. Mov Disord. 2011; 26, 4: 637-643.

[28] Day, B. L., \& Cole, J. Vestibular-evoked postural responses in the absence of somatosensory information. Brain. 2002; 125, 9: 2081-2088.

[29] Mancini, M., Rocchi, L., Horak, F. B., \& Chiari, L. Effects of Parkinson's disease and levodopa on functional limits of stability. Clin Biomech (Bristol, Avon). 2008; 23, 4: 450-458.

[30] Allen, N. E., Canning, C. G., Sherrington, C., \& Fung, V. S. Bradykinesia, muscle weakness and reduced muscle power in Parkinson's disease. Mov Disord. 2009; 24, 9: 1344-1351.

[31] Stack, E., Ashburn, A., \& Jupp, K. Postural instability during reaching tasks in Parkinson's disease. Physiother Res Int. 2005; 10, 3: 146-153.

[32] Suarez H. , Geisinger D. , Ferreira E.D., Nogueira S., Arocena S. , San Roman C., Suarez A. Balance in Parkinson's disease patients changing the visual input. Braz J Otorhinolaryngol. 2011; 77, 5: 651-5.

[33] Jessop R.T., Horowicz C., Dibble L.E. (2006). Motor Learning and Parkinson Disease: Refinement of Movement Velocity and Endpoint Excursion in a Limits of Stability Balance Task. Neurorehabil Neural Repair. 2006; 20: 459-467.

[34] Winter, D. A., Patla, A. E., Prince, F., Ishac, M., \& Gielo-Perczak, K. Stiffness control of balance in quiet standing. J Neurophysiol. 1998; 80, 3: 1211-1221.

[35] Bartolic, A., Pirtosek, Z., Rozman, J., \& Ribaric, S. Postural stability of Parkinson's disease patients is improved by decreasing rigidity. Eur J Neurol. 2005; 12, 2: 156-159.

[36] Horak, F. B., Frank, J., \& Nutt, J. Effects of dopamine on postural control in parkinsonian subjects: scaling, set, and tone. J Neurophysiol. 1996; 75, 6: 2380-2396.

[37] Beckley DJ , Panzer VP, Remler MP, Ilog LB,, Bloem BR. Clinical correlates of motor performance during paced postural tasks in Parkinson's disease. Journal of the Neurological Sciences. 1995; 1, 32, 2: $133-138$ 